\title{
MicroRNA-320 inhibits cell proliferation in glioma by targeting E2F1
}

\author{
JI-YONG SUN ${ }^{*}$, WEI-ZHONG XIAO ${ }^{2 *}$, FEI WANG $^{3}$, YONG-QIAN WANG $^{4}$, \\ YOU-HOU ZHU ${ }^{3}$, YI-FANG WU ${ }^{3}$, ZENG-LI MIAO ${ }^{1}$ and YU-CHANG LIN ${ }^{1}$ \\ ${ }^{1}$ Department of Neurosurgery, Wuxi Second Hospital Affiliated to Nanjing Medical University, Wuxi, Jiangsu 214002; \\ ${ }^{2}$ Department of Neurology, Shanghai Pudong Hospital, Fudan University, Pudong, Shanghai 201399; ${ }^{3}$ Department of \\ Neurosurgery, Tongji Hospital, Tongji University, Shanghai 200065; ${ }^{4}$ Department of Neurosurgery, \\ Longhua Hospital Affiliated Shanghai Traditional Chinese Medical University, \\ Shanghai 200032, P.R. China
}

Received May 4, 2014; Accepted January 9, 2015

DOI: $10.3892 / \mathrm{mmr} .2015 .3657$

\begin{abstract}
MicroRNAs (miRs) are a class of small non-coding RNAs that are involved in the regulation of gene expression, and in cancer development and progression. In the present study, miR-320 expression was found to be significantly reduced in glioma tissue in comparison with that in adjacent healthy tissues. In the present study, in vitro analyses demonstrated that overexpression of miR-320 inhibited cell proliferation and metastasis, while antisense miR-320 oligonucleotides enhanced cell proliferation and migration in U251 and SHG-44 glioma cell lines, compared with that in negative control cells. Protein expression of E2F1, a cell-cycle regulator, was negatively regulated by miR-320. Therefore, the present study provides novel insights into the association between miR-320 and glioma development.
\end{abstract}

\section{Introduction}

Glioma arises from glial cells and accounts for $80 \%$ of all malignant brain tumors (1). Although extensive investigations have been performed in order to understand the mechanisms underlying its pathogenesis, the molecular pathways involved in the initiation and progression of glioma remain unclear (1-3).

MicroRNAs (miRs), a class of non-coding RNA molecules, are involved in the transcriptional and post-transcriptional regulation of gene expression $(4,5)$. Recent investigations have demonstrated that glioma growth is controlled by a number of miRs (6,7). miR-16 inhibits glioma cell growth and inva-

Correspondence to: Dr Fei Wang, Department of Neurosurgery, Tongji Hospital, Tongji University, 389 Xincun Road, Shanghai 200065, P.R. China

E-mail: fei_wangtj@126.com

*Contributed equally

Key words: microRNA-320,E2F1, glioma, metastasis, cell proliferation sion by suppressing the BCL2 and nuclear factor- $\kappa$ B1/MMP9 signaling pathway (8). Other investigations have demonstrated that miR-155 overexpression in patients with glioma is an indicator of poor prognosis (9). Therefore, an investigation into the dysregulated miRs that are involved in the development of glioma may help to elucidate the prognostic value and therapeutic potential of miRs in patients with this disease.

Previous investigations have indicated that miR-320 is involved in the development of a number of types of tumors. For example, miR-320 inhibits cell proliferation and induces apoptosis in breast, prostate and hepatocellular carcinoma (10-13). However, until now, the molecular mechanisms underlying the involvement of miR-320 in gliomagenesis remain poorly understood.

\section{Materials and methods}

Cell culture and tissue samples. U251 and SHG-44 glioma cell lines were obtained from American Type Culture Collection (Rockville, MD, USA). Cells were cultured in Dulbecco's Modified Eagle's Medium(DMEM; Sigma-Aldrich, St. Louis, MO, USA) supplemented with $10 \%$ fetal bovine serum. Cultures were maintained at $37^{\circ} \mathrm{C}$ in a humidified atmosphere with $5 \% \mathrm{CO}_{2}$. Tumor tissues and adjacent healthy tissues were collected during elective therapeutic surgery (tumor resection) at the Department of Neurosurgery, Tongji Hospital (Shanghai, China) between January and March 2014. All samples were obtained with informed consent and with the approval of the institutional review board of Tongji Hospital.

Analysis of miR expression using TaqMan ${ }^{\circledR}$ reverse transcription-quantitative polymerase chain reaction ( $R T-q P C R)$. Total RNA from tissue samples and cell lines was harvested using the miR Isolation kit (Ambion Life Technologies, Carlsbad, CA, USA). Expression of mature miRs was assayed using a TaqMan MiR Assay (Applied Biosystems, Beijing, China) specific for miR-320. Total RNA (5 ng) was reverse transcribed to cDNA using specific stem-loop RT primers (5'-AAAAGC TGGGTTGAGAGGGCGA-3' and a reverse complement primer; Ambion Life Technologies). qPCR was performed 
using Applied Biosystems 7900 Real-Time PCR System (Life Technologies, Shanghai, China) and a TaqMan Universal PCR Master Mix (Life Technologies). Primers were obtained using TaqMan miR Assays. The primer sequences were as follows: Cyclin B1, forward 5'-AATAAGGCGAAGATC AACATGGC-3' and reverse 5'-TTTGTTACCAATGTCCCC AAGAG-3'; Cyclin E, forward 5'-AAGGAGCGGGACACC ATGA-3' and reverse 5'-ACGGTCACGTTTGCCTTCC-3'. Small nuclear U6 RNA (snRNA; Applied Biosystems) was used as an internal control. A total of $50 \mathrm{ng} / \mathrm{ml} \mathrm{cDNA}$ was used in the PCR. PCR conditions included an initial holding period at $94^{\circ} \mathrm{C}$ for $3 \mathrm{~min}$, followed by a two-step PCR program consisting of $95^{\circ} \mathrm{C}$ for $5 \mathrm{sec}$ and $60^{\circ} \mathrm{C}$ for $30 \mathrm{sec}$, for 45 cycles. Relative quantification analysis of gene expression data was performed according to the $2-\Delta \Delta \mathrm{Ct}$ method.

Cell transfection. miR-320 mimics and negative controls (NCs) were obtained from Ambion Life Technologies. In order to inhibit miR-320 expression, an antisense oligonucleotide against miR-320 (Ambion Life Technologies) was used. Lipofectamine $2000^{\circledR}$ (Invitrogen Life Technologies, Carlsbad, CA, USA) and $25 \mathrm{nM}$ of the miRs were prepared according to the manufacturer's instructions. Once the cells had reached $70-80 \%$ confluence they were transfected $\left(2.5 \times 10^{4}\right.$ cells/well $)$ with the mimics, antisense oligonucleotides or NC using Lipofectamine $2000^{\circledR}$, according to the manufacturer's instructions for 24 or $48 \mathrm{~h}$. The same NC was used for the mimic and antisense experiments.

Bromodeoxyuridine (BrdU) assays. A cell proliferation enzyme-linked immunosorbent assay (BrdU kit; Beyotime Biotechnology, Nantong, China) was used in order to assess the incorporation of BrdU during DNA synthesis, according to the manufacturer's instructions. Experiments were repeated three times. Absorbance was measured at $450 \mathrm{~nm}$ using a Spectra Max 190 ELISA reader (Molecular Devices, Sunnyvale, CA, USA)

Cell migration assays. Cell migration capability was analyzed using Transwell ${ }^{\circledR}$ migration chambers (Millipore, Hayward, CA, USA) and a ELx800 absorbance reader (BioTek, Winooski, VT, USA) was used to quantify cell migration at $570 \mathrm{~nm}$, according to the manufacturer's instructions.

Western blotting. Cells or tissues were harvested and lysed with ice-cold lysis buffer (50 mM Tris-HCl, pH 6.8; 100 mM 2-ME; $2 \% \mathrm{w} / \mathrm{v}$ SDS and $10 \%$ glycerol; Beyotime Biotechnology). Following centrifugation at $20,000 \mathrm{x}$ g for $10 \mathrm{~min}$ at $4^{\circ} \mathrm{C}$, proteins in the supernatants were quantified, separated using $10 \%$ SDS PAGE and transferred to a nitrocellulose membrane (Amersham Bioscience, Amersham, UK). Non-fat milk (10\%) in phosphate-buffered saline was used to block the membranes. Subsequently the membranes were immunoblotted using the following primary antibodies: Rabbit polyclonal anti-E2F1 (1:1,000; cat. no. 3742; Cell Signaling Technology, Inc., Beverley, MA, USA) and mouse monoclonal anti-GAPDH (1:10,000; cat. no. sc-365062; Santa Cruz Biotechnology, Inc., Dallas, TX, USA) at $4^{\circ} \mathrm{C}$ for $12 \mathrm{~h}$, followed by horseradish-linked secondary antibodies $(1: 2,000$; cat. nos. 7054 and 7056; Cell Signaling Technology, Inc.) at $25^{\circ} \mathrm{C}$ for $2 \mathrm{hr}$.

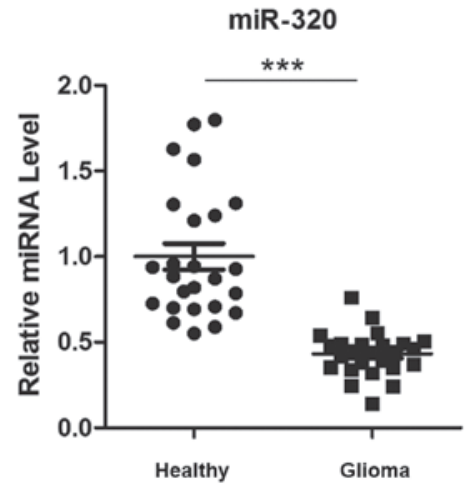

Figure 1. Expression of miR-320 in glioma tissue. miR-320 expression was determined using TaqMan reverse transcription-quantitative polymerase chain reaction in human glioma tissue and adjacent healthy tissue. ${ }^{* * *} \mathrm{P}<0.001$. miRNA, microRNA.

Protein expression was detected using a SuperSignal West Pico Chemiluminescent Substrate kit (Pierce, Rockford, IL, USA) according to the manufacturer's instructions. Anti-E2F1 antibodies were purchased from Cell Signaling Technology, Inc.). Protein expression levels were standardized against GAPDH, using the mouse anti-GAPDH antibody.

Luciferase reporter assay. The potential targets of miR-320 were analyzed using miRWalk software (http://www.umm. uni-heidelberg.de/apps/zmf/mirwalk/) (14). Total cDNA from U251 cells was used to amplify the 3'-untranslated region (UTR) of E2F1. The E2F1 3'-UTR was cloned into an miR expression reporter vector system (pMir-REPORT ${ }^{\mathrm{TM}}$; Ambion Life Technologies). Mutations were introduced into the potential miR-320 binding sites using the QuikChange site-directed mutagenesis kit (Stratagene, Agilent, San Diego, CA, USA). SHG-44 and U251 cells were transfected with miR-320 mimics or NCs using Lipofectamine $2000^{\circledR}$. Subsequently, cells were transfected with the pMir-Report vector or with the mutant construct for $36 \mathrm{~h}$. A pRL-SV40 vector (Promega Corporation, Madison, WI, USA) containing the Renilla luciferase gene was used in order to standardize transfection efficiency. Luciferase activity was determined using the Dual-Luciferase Reporter Assay System (Promega Corporation).

Statistical analysis. Data are expressed as the mean \pm standard error of the mean from at least three repeated experiments. Differences between groups were analyzed using Student's t-test. All statistical analyses were conducted using SPPS version 20.0 (IBM, Armonk, NY, USA) $\mathrm{P}<0.05$ was considered to indicate a statistically significant difference.

\section{Results}

miR-320 expression was downregulated in patients with glioma. In order to analyze the association between miR-320 expression and glioma, miR-320 expression was determined using RT-qPCR in 25 pairs of human glioma tissues and adjacent healthy tissues. Expression of miR-320 was significantly decreased in glioma tissues, compared with that of adjacent healthy tissues (Fig. 1). 
A

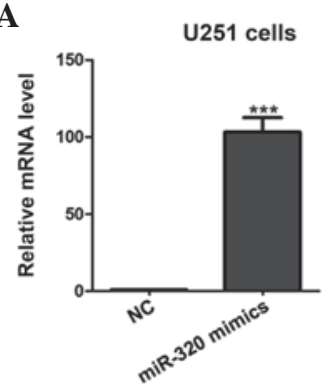

C

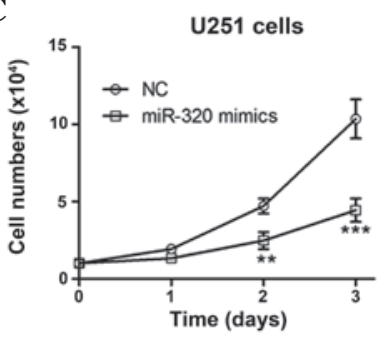

$\mathbf{E}$

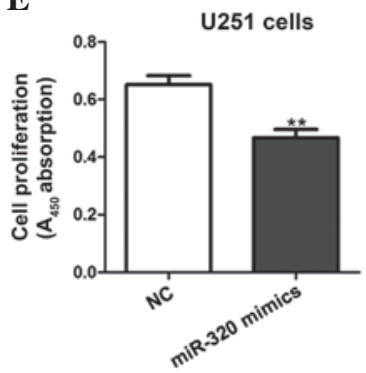

G

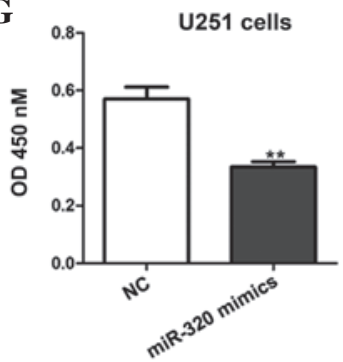

B

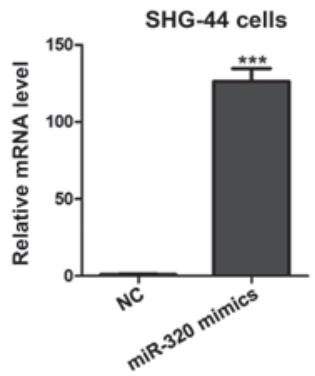

D

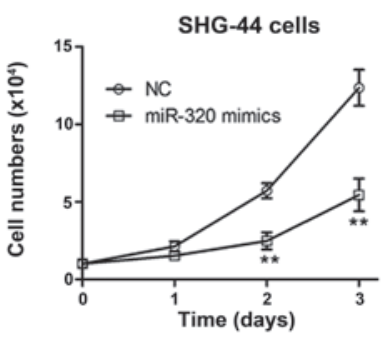

F

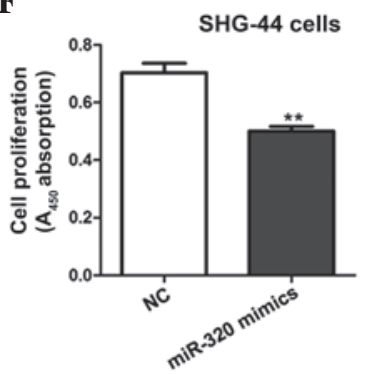

H

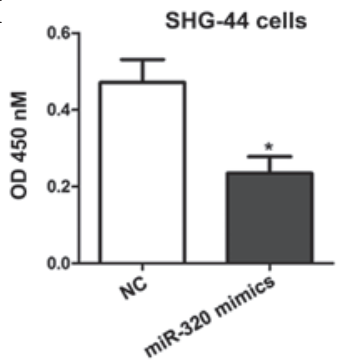

A

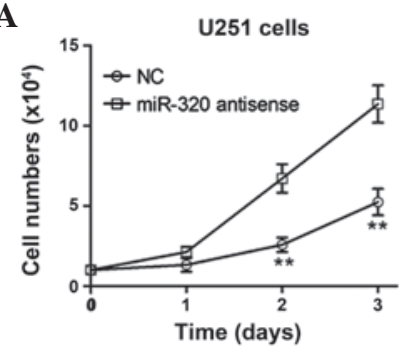

C

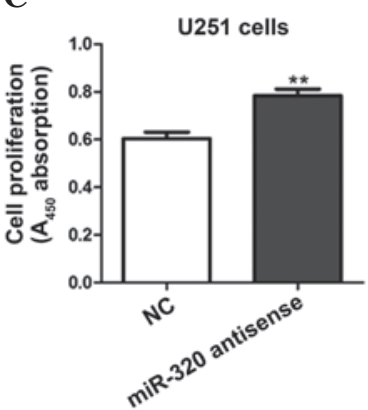

$\mathbf{E}$

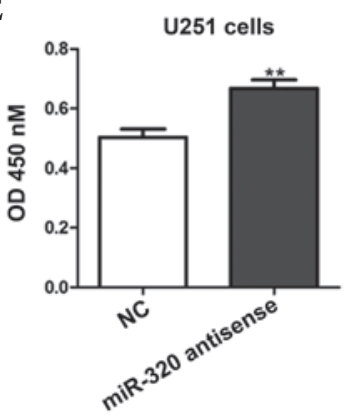

B

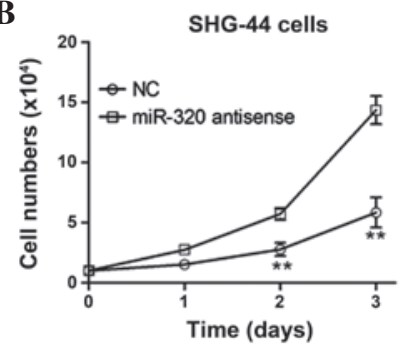

D

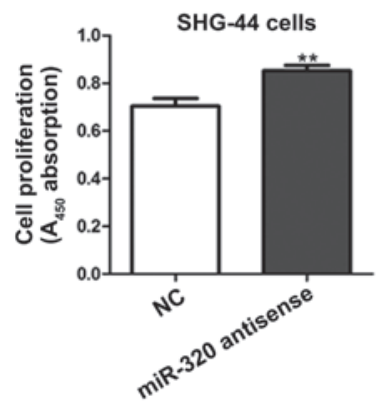

F

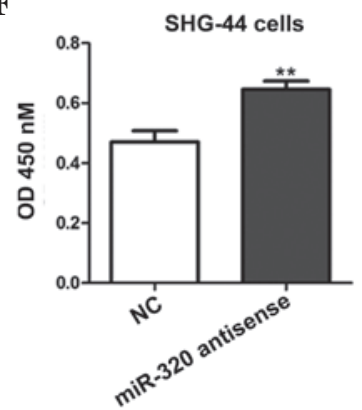

Figure 3. Glioma cell growth following transfection with an antisense oligonucleotide against miR-320 or with NC. (A and B) Growth curve of U251 and SHG-44 cells. (C and D) Cell proliferation in U251 and SHG-44 cells. A450 absorption was assayed following transfection for $36 \mathrm{hr}$. (E and F) Transwell migration assays were conducted in $\mathrm{U} 251$ and $\mathrm{SHG}-44$ cells. ${ }^{*} \mathrm{P}<0.05$, ${ }^{*} \mathrm{P}<0.01,{ }^{*} \mathrm{P}<0.001$, as compared with NC. miR, microRNA; NC, negative control; OD, optical density.

Figure 2. miR-320 overexpression inhibited glioma cell growth following transfection with miR-320 mimic or NC. (A and B) Expression of miR-320 in U251 and SHG-44 glioma cell lines. (C and D) Growth curves of U251 and SHG-44 cells. (E and F) U251 and SHG-44 cell proliferation. A450 absorption was assayed following transfection with an miR-320 mimic or $\mathrm{NC}$ for $36 \mathrm{hr}$. (G and H) Following transfection with miR-320 mimic or NC for 36 hours, U251 and SHG-44 cells were seeded in transwell filters and incubated for 24 h. ${ }^{*} \mathrm{P}<0.05,{ }^{*} \mathrm{P}<0.01,{ }^{*} \mathrm{P}<0.001$, as compared with NC. NC, negative control; miR, microRNA; OD, optical density.

miR-320 overexpression inhibits cell proliferation. In order to assess the effects of miR-320 on glioma cell growth, miR-320 mimics or NCs were transfected into U251 and SHG-44 cells (Fig. 2A and B). In comparison with NC cells, cell numbers and proliferation were significantly lower in U251 and SHG-44 cells lines overexpressing miR-320 mimics (Fig. 2C-F). Furthermore, miR-320 mimic overexpression significantly inhibited in vitro migration capabilities of the U251 and SHG-44 cell lines compared with that of the NC cells (Fig. 2G-H).

Inhibition of miR-320 promotes the proliferation of glioma cells. In order to demonstrate the association between miR-320

expression and glioma cell growth, U251 and SHG-44 cells were transfected with an antisense oligonucleotide against miR-320, which inhibited endogenous miR-320 activity. This resulted in enhanced growth of the antisense-transfected cells compared with that of NC cells (Fig. 3A-D). In addition, the in vitro migration capability of antisense-transfected cells was significantly higher than that of NC cells (Fig. 3E-F).

miR-320 directly targets the E2F1 in glioma cells. Using a bioinformatics approach (miRWalk software), a number of putative target genes of miR-320 were identified (data not provided), among which the gene encoding E2F1 harbored a potential miR-320 binding site (Fig. 4A). Luciferase activity was significantly lower in cells overexpressing miR-320 compared with NC cells following transfection with pMir-REPORT vectors (Fig. 4B). No significant difference in luciferase activity was observed in mutated U251 and SHG-44 cells (mutation of the conserved miR-320 binding motif) between cells overexpressing miR-320 and NC cells (Fig. 4B). E2F1 protein expression was lower in cells overexpressing miR-320 mimics compared with that of the NC cells (Fig. 4C-D). 
$\mathbf{A}$

B

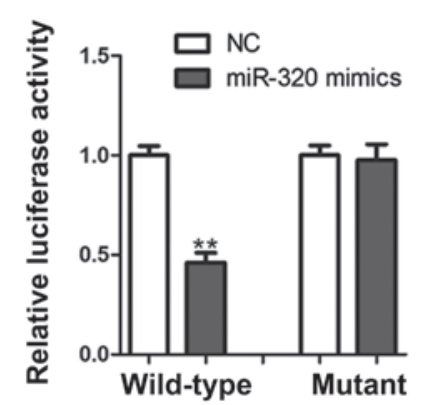

C

\section{U251 Cells}

NC miR-320 mimics

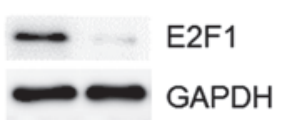

D

SHG-44 Cells

NC miR-320 mimics

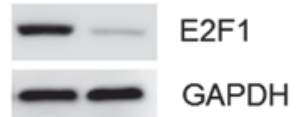

$\mathbf{E}$

\section{U251 Cells}

NC miR-320 antisenss

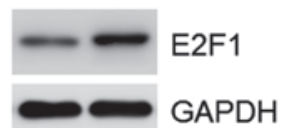

$\mathbf{F}$

\section{SHG-44 Cells}

NC miR-320 antisense

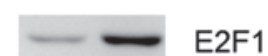

G

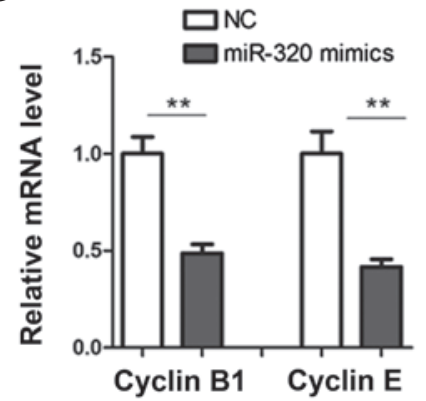

$\mathbf{H}$

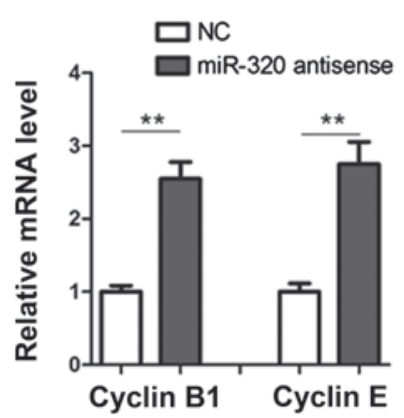

Figure 4. miR-320 regulates E2F1 expression in glioma cells. (A) Computer prediction of miR-320 binding sites in the 3'UTRs of human E2F1 genes. A potential binding site is highlighted in bold. (B) Luciferase reporter assays in U251 cells. Cells were transfected with 100 ng of wild-type 3'-UTR-reporter or mutant constructs with $100 \mathrm{nM}$ of the miR-320 mimic or NC. (C and D) Representative western blots of E2F1 in U251 and SHG-44 cells following transfection with miR-320 mimic or NC. (E and F) Western blotting of E2F1 in U251 and SHG-44 cells following transfection with miR-320-antisense or NC. (G) mRNA expression of Cyclin B1 and Cyclin E in U251 cells following transfection with miR-320 mimics or NC. (H) mRNA expression of Cyclin B1 and Cyclin E in U251 cells following transfection with miR-320 antisense or NC. ${ }^{* *}$ p $<0.01$, miR-320 antisense vs. NC. miR, microRNA; NC, negative control; UTR, untranslated region.

A

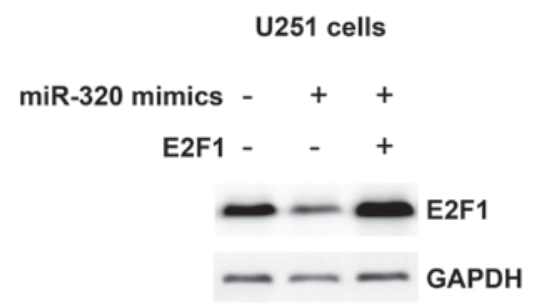

$\mathbf{C}$

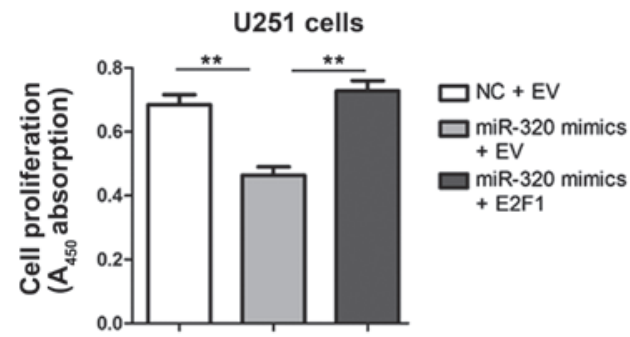

B

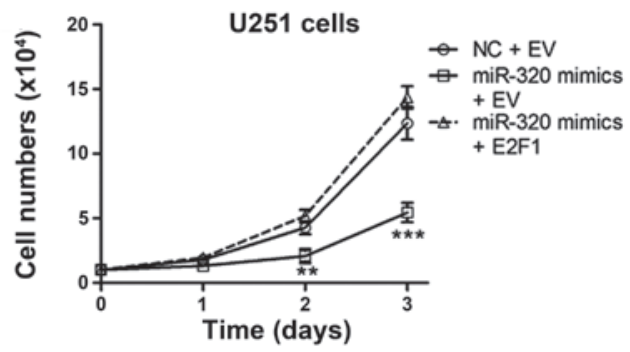

D

U251 cells

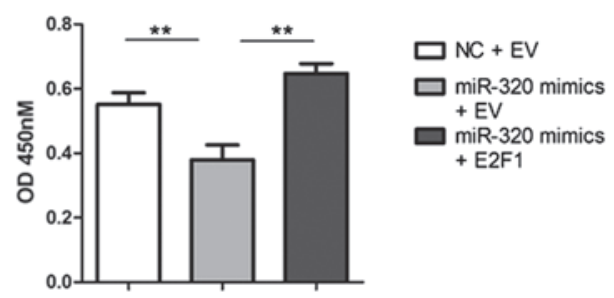

Figure 5. The anti-proliferative affect of miR-320 is associated with E2F1. (A) E2F1 protein expression using western blotting in U251 cells transfected with miR-320 mimics or NC, followed by transfection with lentiviruses containing the E2F1 gene or EV.(B) U251 cell growth curve and (C) U251 cell proliferative potential. (D) Transwell migration assays were conducted in U251 cells. miR, microRNA; NC, negative control; EV, empty vector; OD, optical density. 
E2F1 protein expression was significantly higher in antisense miR-320-transfected cells compared with that in the NC cells (Fig. 4E-F), which indicates that E2F1 may be a target of miR-320 in glioma cells. Expression levels of down-stream target genes of E2F1, Cyclin B1 and Cyclin E $(13,15)$, were significantly higher in cells overexpressing miR-320 mimics and significantly lower in antisense miR-320-transfected cells compared with those of the NC cells in each case (Fig. 4G-H).

In order to verify the association between miR-320 and E2F1, U251 cells were transfected with lentiviruses containing an E2F1 gene or an empty vector (EV), following transfection with miR-320 mimics (Fig. 5A). U251 cell proliferation and migration levels were significantly lower following transfection with miR-mimic and the EVs compared with those of cells transfected with miR-320 mimic and E2F1 vectors, and with NC cells transfected with EVs (Fig. 5B-D). Overall, the results of the present study suggest that the mechanisms underlying the effect of miR-320 on glioma development are associated with the regulation of $\mathrm{E} 2 \mathrm{~F} 1$.

\section{Discussion}

A recent study demonstrated that miR-320 regulates tumor angiogenesis, which is driven by vascular endothelial cells in oral cancer by silencing the expression of neuropilin 1 (16). Another study demonstrated that miR-320 inhibits osteosarcoma cell proliferation by directly targeting fatty acid synthase (17). In the present study, miR-320 expression was shown to be lower in glioma tissues compared with that of adjacent healthy tissues. The results suggest that miR-320 overexpression inhibits glioma cell proliferation, metastasis and invasion, compared with that of NC cells and miR-320 antisense-transfected cells. Therefore, in accordance with previous reports, the present study suggests that miR-320 may suppress tumor development and progression.

Computational algorithms have been used to predict multiple genes as potential targets of miR-320 $(16,17)$. The results of the present study suggest that miR-320 targets the 3'UTR of E2F1 and is involved in regulating E2F1 expression. $\mathrm{E} 2 \mathrm{~F} 1$ is a transcription factor involved in the $\mathrm{pRb} / \mathrm{E} 2 \mathrm{~F} 1$ pathway and in the regulation of the G1/S phase (18). Tumor suppression triggered by $\mathrm{pRb}$ has been associated with its ability to inhibit E2F1-responsive promoters (18). E2F1-responsive promoters were more active in glioma cells than in healthy cells in an investigation by Alonso et al (19), which suggests that there is more 'free' E2F1 and fewer $\mathrm{pRb} / \mathrm{E} 2 \mathrm{~F} 1$ repressor complexes in glioma cells compared with healthy cells. E2F1 activity is capable of suppressing and promoting tumor development (20). Although the precise mechanisms are currently unclear, E2F1 activity may be dependent on the nature of associated oncogenic mutations. A cluster of miRs, including miR-106a, miR-138 and miR-329, promoted glioma cell proliferation by targeting E2F1 (21-23), which suggests that E2F1 is involved in glioma cell proliferation and that $\mathrm{E} 2 \mathrm{~F} 1$ expression may be regulated by a number of factors.

In conclusion, the present study demonstrated that miR-320 was downregulated in glioma tissues and could inhibit cell proliferation by targeting E2F1. Since E2F1 has a critical role in cell proliferation, differentiation and apoptosis, further studies are required to establish the role of miR-320 in glioma.

\section{References}

1. Goodenberger ML and Jenkins RB: Genetics of adult glioma. Cancer Genet 205: 613-621, 2012.

2. Weller $M$ and Wick W: Neuro-oncology in 2013: improving outcome in newly diagnosed malignant glioma. Nat Rev Neurol 10: 68-70, 2014.

3. Castro MG and Lowenstein PR: Neuro-oncology: The long and winding road - gene therapy for glioma. Nat Rev Neurol 9: 609-610, 2013.

4. Sun K and Lai EC: Adult-specific functions of animal microRNAs. Nat Rev Genet 14: 535-548, 2013.

5. Ameres SL and Zamore PD: Diversifying microRNA sequence and function. Nat Rev Mol Cell Biol 14: 475-488, 2013.

6. Auffinger B, Thaci B, Ahmed A, Ulasov I and Lesniak MS: MicroRNA targeting as a therapeutic strategy against glioma. Curr Mol Med 13: 535-542, 2013.

7. Palumbo S, Miracco C, Pirtoli L and Comincini S: Emerging roles of microRNA in modulating cell-death processes in malignant glioma. J Cell Physiol 229: 277-286, 2014.

8. Yang TQ, Lu XJ, Wu TF, et al: MicroRNA-16 inhibits glioma cell growth and invasion through suppression of BCL2 and the nuclear factor- $\kappa \mathrm{B} 1 / \mathrm{MMP} 9$ signaling pathway. Cancer Sci 105: 265-271, 2014.

9. Sun J, Shi H, Lai N, Liao K, Zhang S and Lu X: Overexpression of microRNA-155 predicts poor prognosis in glioma patients. Med Oncol 31: 911, 2014.

10. Bronisz A, Godlewski J, Wallace JA, et al: Reprogramming of the tumour microenvironment by stromal PTEN-regulated miR-320. Nat Cell Biol 14: 159-167, 2011.

11. Hsieh IS, Chang KC, Tsai YT, et al: MicroRNA-320 suppresses the stem cell-like characteristics of prostate cancer cells by downregulating the Wnt/beta-catenin signaling pathway. Carcinogenesis 34: 530-538, 2013.

12. Yao J, Liang LH, Zhang Y, et al: GNAI1 suppresses tumor cell migration and invasion and is post-transcriptionally regulated by Mir-320a/c/d in hepatocellular carcinoma. Cancer Biol Med 9: 234-241, 2012.

13. Engelmann D and Pützer BM: The dark side of E2F1: in transit beyond apoptosis. Cancer Res 72: 571-575, 2012.

14. Dweep H, Sticht C, Pandey P and Gretz N: miRWalk - database: Prediction of possible miRNA binding sites by "walking" the genes of three genomes. J Biomed Inform 44: 839-847, 2011.

15. Biswas AK and Johnson DG: Transcriptional and nontranscriptional functions of E2F1 in response to DNA damage. Cancer Res 72: 13-17, 2012.

16. Wu YY, Chen YL, Jao YC, Hsieh IS, Chang KC and Hong TM: miR-320 regulates tumor angiogenesis driven by vascular endothelial cells in oral cancer by silencing neuropilin 1 . Angiogenesis 17: 247-260, 2014.

17. Cheng C, Chen ZQ and Shi XT: MicroRNA-320 inhibits osteosarcoma cells proliferation by directly targeting fatty acid synthase. Tumour Biol 35: 4177-4183, 2014.

18. Pützer BM and Engelmann D: E2F1 apoptosis counterattacked: evil strikes back. Trends Mol Med 19: 89-98, 2013.

19. Alonso MM, Alemany R, Fueyo J and Gomez-Manzano C: E2F1 in gliomas: a paradigm of oncogene addiction. Cancer Lett 263: $157-163,2008$.

20. Fueyo J, Gomez-Manzano C, Liu TJ and Yung WK: Delivery of cell cycle genes to block astrocytoma growth. J Neurooncol 51: 277-287, 2001.

21. Yang G, Zhang R, Chen X, et al: MiR-106a inhibits glioma cell growth by targeting E2F1 independent of p53 status. J Mol Med (Berl) 89: 1037-1050, 2011.

22. Qiu S, Huang D, Yin D, et al: Suppression of tumorigenicity by microRNA-138 through inhibition of EZH2-CDK4/6-pRb-E2F1 signal loop in glioblastoma multiforme. Biochim Biophys Acta 1832: 1697-1707, 2013.

23. Xiao B, Tan L, He B, Liu Z and Xu R: MiRNA-329 targeting E2F1 inhibits cell proliferation in glioma cells. J Transl Med 11: 172, 2013. 